\title{
Multiple epiphyseal dysplasia due to collagen 9 anomaly
}

INSERM

\section{Source}

INSERM. (1999). Orphanet: an online rare disease and orphan drug data base. Multiple epiphyseal dysplasia due to collagen 9 anomaly. ORPHA:166002

Multiple epiphyseal dysplasia due to collagen 9 anomaly is a rare primary bone dysplasia disorder characterized by normal or mild short stature, early-onset pain and/or stiffness of the joints (mainly affecting knees but also elbows, wrists, ankles and fingers, with relative sparing of the hips) and early degenerative joint disease. Other skeletal anomalies (incl. varus or valgus deformities, osteochondritis dissecans, abnormal carpal shape, free articular bodies) and mild myopathy have also been reported. 